\title{
Effect of Unipolar Magnetic Field on Macroscopic Properties of Distilled Water
}

\author{
Valery Shalatonin \\ State University of Informatics and Radioelectronics, Minsk, Belarus \\ Email: shalatonin@bsuir.by
}

\begin{abstract}
Differences in biophysical properties of water $(\mathrm{pH}$ temperature, evaporation and bioactivity) affected by the north and south polarity magnetic field are studied. The magnetic field (MF) is produced by a ring-shaped permanent magnet. The intensities of the $M F$ at the extremities from the center are in the range of $45-65 \mathrm{mT}$. Petri dish (PD) containing distilled water was left on the surface of this magnet ( $\mathrm{N}$ or $\mathrm{S}$ pole) for a long period of time (up to 70 hours). It is shown that the changes in $\mathrm{pH}$ and in amount of evaporated water have the opposite character for different poles of the magnet in comparison with water in the control PD. It is found that the MF decreases the water evaporation and $\mathrm{pH}$ in case of the south polarity $\mathrm{MF}$ and increases the amount of evaporated water and $\mathrm{pH}$ if the north polarity MF is applied. The temperature of the distilled water treated by the MF gets higher $\left(0.4-0.8{ }^{\circ} \mathrm{C}\right)$ in comparison with the water in the control PD.
\end{abstract}

Index Terms - water, magnetic field, north and south polarity, $\mathrm{pH}$, evaporation, temperature

\section{INTRODUCTION}

Water is one of the most abundant constituents of the universe and is composed of hydrogen and oxygen, the first and third most abundant elements in the universe, respectively. The fact that water was present long before the evolution of life on earth suggests that its unique properties have strongly conditioned life as we know it. The water content of an adult human is as high as $65-70 \%$. Water promotes the formation of biological structures, enables biological hydrolysis, and acts as a solvent distributing nutrients and removing metabolism products [1], [2]. The research of Pollack et al. [3]-[5] suggests that, in addition to solid, liquid and gaseous state, there is also a fourth aggregate state of water, namely the liquid crystalline phase. It was shown that water molecules are ordered in some few hundred thousand layers away from hydrophilic gels. The water forms massive exclusion zones (EZs) completely (or almost completely) without solutes next to the surfaces of hydrophilic gels. The clear zone next to the gel surface was a few hundred microns deep and stayed stable for days and even weeks.

As water is the main medium where the major part of biochemical reactions is taking place, it is supposed that the environmental exposures to natural and artificial electromagnetic fields may change metabolic activity of

Manuscript received March 5, 2017; revised July 2, 2017 cells and organisms using body's water as a primary receptor [6]. Although the structural and dynamical alterations are subtle, they can perturb a well-balanced system sufficiently to facilitate disease. The disruption of water dynamics between and within cells underlies many disease conditions [7]. The search for a biological effect due to magnetic fields has a long history dating back a hundred years [8]-[10].

As was mentioned above the water is probably the primary receptor of the MF in biological systems. The phenomenon of liquid water treatment with an applied magnetic field has been known for many years. The obtained results largely confirm this assumption.

In Ref. [11], [12] the infrared adsorption spectrum and Raman spectrum of magnetized water were analysed. It was found that static magnetic field causes changes in the distribution and polarization of the molecules of water. This was deduced from an increase in some of the peaks strength, shift in their frequencies, as well as appearance of some additional peaks. The authors noted that the magnetized water really has magnetism, which has been verified by a peak shift of X-ray diffraction of magnetized water. These effects were related to the time of magnetization, the intensity of applied MF and the temperature of water. It was also found that the magnetic fields reduce the specific heat of water, increase the soaking degree and hydrophobicity of water to materials, depress its surface tension force, and increase the refraction index, dielectric constant and electrical conductivity of water. It was alleged that these changes were caused by the above changes of the microscopic structures under the action of magnetic field.

Magnetic treatment of liquid water treatment at static and flow conditions has been used in numerous applications, such as water purification, drug delivery, microbial degradation of environmental pollutants, sterilization, agriculture etc. Magnetic water controls scale buildup in pipes and plumbing [13]. In spite of those practical applications, the underlying physical mechanisms responsible for the changes in the properties of water have not yet been fully understood.

Our studies have been focused on the effect of a unipolar magnetic field on the physical and chemical properties of distilled water. It is experimentally shown that there are fundamental differences in the biophysical effects of the MF of the north and south polarity. Some of studies found that each pole of a magnet has specific 
effects that are quite different [14]-[21]. In Ref. [14] first discovered that the north and south poles of all magnets exhibit unique and different properties with respect to their effects on various forms of life. It was shown that the south pole of a magnet when placed near a living organism has a positive, enhancing effect, while the north pole - has a negative, retarding effect. The authors presented numerous examples of enhancement to seeds and various types of cellular growth. However, these studies have not been confirmed by some other researchers and currently are not generally accepted. More recent experimental evidence suggests that the interaction of MF with microorganisms is directionally dependent. The effect of magnetic fields on the rate of phenol biodegradation using immobilized activated sludge was studied in [15], [16]. This study was conducted by applying separately the north pole and the south pole magnetic fields to the bioreactor. Rate of dissolved oxygen consumption, phenol concentration and extracellular protein concentration were the parameters monitored during the process. It was observed that by applying a magnetic south pole to the process, biodegradation in the form of biological oxidation was enhanced. Microbial pellets in the presence of south pole magnetic fields demonstrate enhanced oxidation of phenol. A 30\% increase in biodegradation rate was obtained by applying a magnetic south pole of strength of $0.45 \mathrm{~T}$ to the bioreactor with immobilized microbial beads as compared to the control. Maximum observed enhancement with twice irradiated cells was $750 \%$ higher than the control run with no magnetic field. Magnetic north pole irradiation inhibited this type of biooxidation. This process has potential for biological treatment of organic wastes.

In Ref. [17] was found that Streptococcus mutans bacteria showed a 50 to $60 \%$ increase in accumulation on the geomagnetic south side of a glass surface compared with the north side. Reversal of the direction of the magnetic field by 180 degrees produces a similar reversal in the direction of the preferential accumulation. These results are in accordance with data obtained recently [18]. Plants grown in Petri plates were exposed to both normal and reversed geomagnetic field (GMF) conditions. The data show for the first time that reversing the GMF polarity has significant effects on plant growth and gene expression. This supports the hypothesis that GMF reversal contributes to inducing changes in plant development that might justify a higher selective pressure, eventually leading to plant evolution.

In Ref. [19] the effect of a weak permanent magnetic field (PMF) with strength of $62 \mu \mathrm{T}$ on chilli (Capsium annum L.) for seed germination, growth and development was studied. The authors were carried out the experiment to compare the four pre-sowing treatments at different times $(4,8,12$ and $24 \mathrm{~h}$ exposure) of chilli seeds with the untreated control. Statistically processed experimental data suggest that the effect of static MF significantly increased the seed germination and initial development. The seeds treated with south pole were showing maximum growth status as compared to north pole and untreated control. Both magnetic poles with different treatment times reveal increases in germination speed and total length of plants.

Important results were obtained in Ref. [20]. It was shown that unidirectional magnetic fields inhibited (north pole) or increased (south pole) the growth of cancer cells. An experiment was performed using human lung carcinoma cells. The latest results show that static MF can inhibit the proliferation of cancer cells, and the killing effects of static MF combined with antineoplastic drugs on cancer cells are greater than those of static MFs or anticancer drugs alone. These observations suggest a potential strategy for chemotherapy, that is, the combination therapy of MFs and chemotherapeutic drugs. However, so far it remains unclear which mechanism underlies the killing effects of static MFs combined with chemotherapy drugs on cancer cells [21], [22]. Unfortunately, in these studies did not pay any special attention to the MFs polar properties.

There are evidences that the biological effects of MFs are connected to an alteration in the structure of water that impedes the flux of protons in adenosine triphosphate synthase (ATPS) hydrophilic channels. These results may be environmentally important, in view of the central role played in human physiology by ATPS, particularly in the possible link to diabetes, cancer and longevity [23].

It should be noted, that in some other studies that have focused on the biological effects of a unipolar magnetic field on human tissue no different effects from the north or south poles are found. For example, the results of the study [24] demonstrated no difference in heart rate (HR) and blood pressure (BP) for three groups of people which were influenced by the north, south and sham magnetic field. During experiment people were laid on a mattress containing magnets $(40-50 \mathrm{mT})$. It was concluded that short-term exposure (up to 15 minutes) the MF of either polarity does not appear to cause any clinically meaningful changes in HR or BP among asymptomatic subjects. In [25] was investigated the localized effect of the different magnetic poles and the sham field over the palmer part of the second finger for 15 minutes. Measurements on skin microcirculation for three treatment groups of people were made and showed a significant reduction in blood circulation for both sides of the magnet when compared to the sham field but there was no significant difference between MF of the north and south poles.

There is one more interesting phenomenon possibly related to the problem of the unipolar MF. The geomagnetic field is an important natural environmental factor for life on the Earth. The poles of that field are located not far from the Earth's geographic poles. Its strength at the Earth's surface ranges from less than 30 $\mu \mathrm{T}$ (South America and South Africa) to almost $70 \mu \mathrm{T}$ around the magnetic poles in northern Canada and south of Australia, and in part of Siberia [26]. The GMF is steadily acting on living systems and influences many biological processes. According to religious and cultural beliefs sleeping with head in different directions has different effects on sleep quality of people. In particular, 
it is stated that one must never keep his head in the North geographical direction while sleeping.

It was studied in [27] whether head direction has any effect on heart rate (HR), blood pressure (BP) and serum cortisol (SC) during supine rest. On comparing the parameters in different directions it was observed that those people who were instructed to sleep with head in the South geographical direction (or the north magnetic direction of the Earth) for 12 weeks had lowest systolic and diastolic $\mathrm{BP}, \mathrm{HR}$ and $\mathrm{SC}$ which were found to be statistically significant. These results would seem to contradict the conclusions in [24] where the induction of the MF used was about 1000 times more. It is possible that the clinically meaningful changes in $\mathrm{HR}$ and $\mathrm{BP}$ could be caused only a long-term MF exposure even if the induction is low.

As can be seen from the above, further studies are needed to understand the differences in biophysical effects caused by unipolar magnetic fields and mechanisms of their appearance.

This article examines the effect of a unipolar MF on the macroscopic properties of liquid water. It is shown that there are fundamental differences in properties of the MF of the north and south polarity. The MF decreases the water evaporation and $\mathrm{pH}$ in case of the south polarity and increases the amount of evaporated water and $\mathrm{pH}$ if the north polarity $\mathrm{MF}$ is applied. The unipolar MF increases temperature of distilled water.

\section{OBJECTS AND METHODS}

The experimental design was performed under laboratory conditions, at a mean room temperature of 22 $\pm 2{ }^{\circ} \mathrm{C}$. The $\mathrm{MF}$ is produced by the permanent magnet which is a ferromagnetic disc $50 \mathrm{~mm}$ thick, $135 \mathrm{~mm}$ in diameter with a hole of $57 \mathrm{~mm}$ in diameter. The intensities of the magnetic field at the extremities from the centre were in the range of 45-65 mT. For the magnetic treatment a Petri dish (PD) containing distilled water $(30 \mathrm{ml})$ was left on the surface of this magnet $(\mathrm{N}$ or $\mathrm{S}$ pole) for a long period of time (up to 70 hours). The control PD and the PD influenced by the magnet were covered with equal sheets of paper (Fig. 1). The water was influenced by the unipolar static MF of the magnet for a long period of time (up to 70 hours). The comparative experiments with different poles of the magnet had been completed under the same conditions. For our measurements a $\mathrm{pH} / \mathrm{ORP} /{ }^{\circ} \mathrm{C}$-meter HI 8314 with accuracy $\pm 0.01 / \pm 1 / \pm 0.4$ was used. Volumetric flasks were used to measure a volume of the water. The grains of wheat were used as test objects in order to determine whether there are differences in bioactivity of the magnet poles. Two PD with grains (generally 100 grains) were put on the surface of additional parallelepiped-shaped $(84 \times 64 \times 14 \mathrm{~mm})$ magnets $(\mathrm{N}$ and $\mathrm{S}$ poles). They have the magnetic field induction in the range of 45-100 $\mathrm{mT}$. The quantity of the grains in each dish and its watering remained the same throughout the experiment. All the experiments were stopped by the 9 th day of seeding.

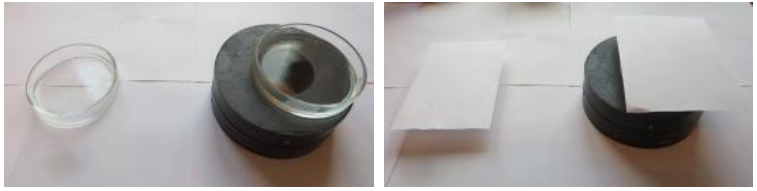

Figure 1. Disposition of the magnet and Petri dishes with water.

\section{EXPERIMENTAL RESULTS}

The main experimental results in Table I and in Fig. 2 and Fig. 3 are shown. The relative changes in parameters $\mathrm{pH}, \mathrm{V}$, and $\mathrm{T}$ are calculated by using the following formulas: $\delta \mathrm{pH}=\left(\mathrm{pH}_{\mathrm{m}}-\mathrm{pH}_{\mathrm{c}}\right) / \mathrm{pH}_{\mathrm{c}}, \delta \mathrm{V}=\left(\mathrm{V}_{\mathrm{m}}-\mathrm{V}_{\mathrm{c}}\right) / \mathrm{V}_{\mathrm{c}}, \delta \mathrm{T}$ $=\left(\mathrm{T}_{\mathrm{m}}-\mathrm{T}_{\mathrm{c}}\right) / \mathrm{T}_{\mathrm{c}}$, where $\mathrm{pH}_{\mathrm{c}}\left(\mathrm{pH}_{\mathrm{m}}\right), \mathrm{V}_{\mathrm{c}}\left(\mathrm{V}_{\mathrm{m}}\right)$ and $\mathrm{T}_{\mathrm{c}}\left(\mathrm{T}_{\mathrm{m}}\right)$ are numerical values of $\mathrm{pH}$, volume of water in the $\mathrm{PD}$ and the temperature of the distilled water for control (magnetized) water respectively.

TABLE I. EFFECT OF MAGNETIC FIELD ON PROPERTIES OF WATER

\begin{tabular}{|c|c|c|}
\hline \multirow{2}{*}{ Parameter } & \multicolumn{2}{|c|}{ Magnetic field } \\
\cline { 2 - 3 } & North pole & $\begin{array}{c}\text { South } \\
\text { pole }\end{array}$ \\
\hline $\mathrm{pH}$ of water (curve 1) & increases & decreases \\
\hline $\begin{array}{c}\text { Amount of evaporated water } \\
\text { (curve 2) }\end{array}$ & increases & decreases \\
\hline $\begin{array}{c}\text { Temperature of water (curve 3) } \\
\text { increases }\end{array}$ & increases \\
\hline
\end{tabular}

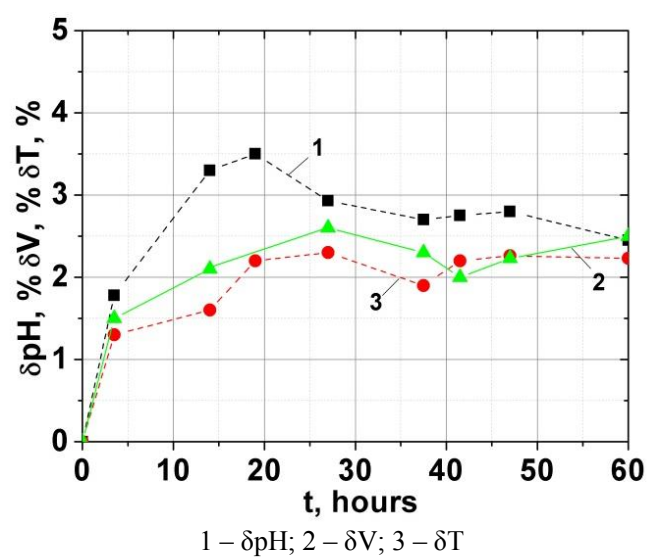

Figure 2. Experimental results for the North pole.

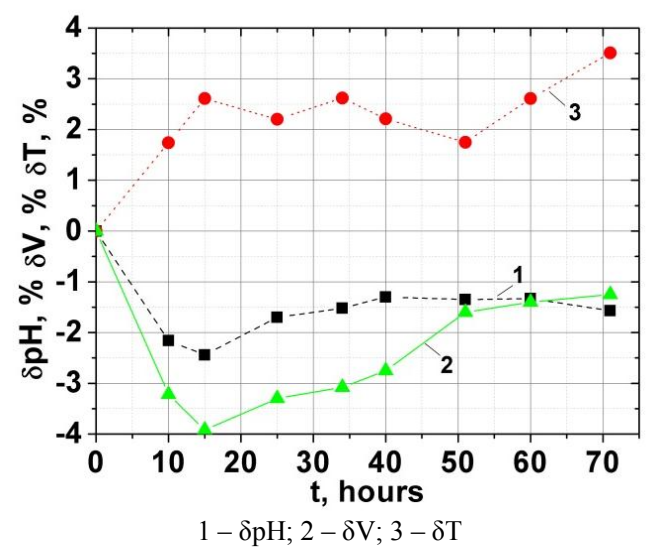

Figure 3. Experimental results for the South pole. 
From the above, it can be seen that the MF of the north and south poles of the magnet have the opposite effect on the $\mathrm{pH}$ and the water vaporization. The impact of the MF of the north and south poles leads to a slight alkalization and oxidation of water, respectively. These results do not contradict existing experimental results [14]-[20]. Furthermore, they are in close agreement with the data reported in [15], [16].

It was also found that the north pole MF increases and the south pole MF decreases the amount of evaporated water. The influence of a bipolar magnetic field on the water vaporization rate has been investigated in several publications [28]-[30]. It was shown that even weak magnetic fields $(15 \mathrm{mT})$ can increase the evaporation rate of water. Various explanations for this phenomenon have been given. An enhancement of evaporation demonstrated that hydrogen bonding may be weakened or even broken by the forces of the magnetic field. The weakening of hydrogen bonding and van der Waals forces between water molecules caused by a magnetic field makes it easier for water molecules to escape from the surface [28]. The amount of water evaporated is essential depends on the properties of a water/air interface and the field gradients at different positions of the water in the magnetic field [30].

As can be seen in Fig. 1 and Fig. 2 (curves 3) the exposure to the unipolar MF leads to the water temperature increase. A simple search of the literature shows that this effect is known very little. Nevertheless, it has been shown in [31] that the water temperature increases in process of its cycling treatment by a magnetic field $(40 \mathrm{mT})$. Thermal images of the aqueous surface layer demonstrate a non-uniform temperature distribution before and after the magnetic field cycling. The increase in the number of cycles provides the temperature increase; the difference is $1-2^{\circ} \mathrm{C}$ as compared to the test objects. In the authors' opinion, this effect can be explained by the dispersion of large water clusters after impact of magnetic field cycles. The small formed fragments possess mobility higher than the original associates. Therefore, the dispersion of water clusters in the magnetic field is also highly probable. As consequence, the increase of the aqueous medium temperature is observed.

It should be noted that the effects of the magnetic field on hydrogen bonds and van der Waals forces were earlier discussed, but have not yet been verified due to contradictory reports [30].

As was mentioned above the water is probably the primary receptor of the MF in biological systems. It means that different changes in properties of water under exposure to the MF of the north or south poles may change differently the metabolic activity of plants. The grains of wheat were used as test objects in order to determine whether there are differences in bioactivity of the magnet poles. The obtained results agree with the positive effects of the MF on development of plants known from literature. Both magnetic poles reveal growth the length and mass of wheat sprouts. Nevertheless, seeds treated with the south pole show maximum positive influence of the MF: number of shoots and total mass of sprouts are increased (10-20\% and $15-$ $26 \%$ respectively), that correlates with the results obtained in [19]. It is known that wheat grows better on slightly acid soil. It is probably means that the water treated with the MF of the south pole really becomes more acidic. This is consistent with the results of our experiments (Fig. 2, curve 1). It is interesting that the north pole influence shown little decrease in number of shoots (up to $-10 \%$ ) but in spite of that, the total mass of sprouts gets bigger $(5-10 \%)$ in comparison with the control group. The detailed report of the study will be published later.

\section{CONCLUSION}

The experiments showed that there are principal differences in physical properties of distilled water under the influence of unipolar magnetic field ( $\mathrm{N}$ or $\mathrm{S}$ pole). The obtained results consistent well with the other studies where the different effects of the MF both poles were examined.

Therefore, additional investigations of the effects of the unipolar magnetic field on macroscopic properties of distilled water are still needed to further clarify the phenomena and its related mechanisms.

At present most of the proposed ideas devoted to this problem are being based on the on the molecular level of water and closely connected with the water clustering. But these results cannot explain how the non-thermal magnetic and EM fields could essentially affect the hydrogen bonds in water during clustering. According to [32] — . . the detailed molecular-level properties of water are very important. Yet, it is becoming evident that they are not sufficient to provide a complete theory of water; we are missing some essential water aspects that cannot be accounted for by the molecular level investigations irrespective of how detailed and sophisticated they will be". We suppose that we are missing not only some essential water aspects but we are also missing essential properties of the magnetic field and EM radiation, without which it is impossible to explain the phenomenon of water.

Thus, our interpretation of the transition of water treated by a MF from an ordinary state to a new one is mainly related to the intrinsic properties of the hydrogen protons and probably the protons and neutrons of oxygen in molecules of water. New physical state of water changes its macroscopic and, therefore, biological properties [33].

Insight into the described phenomena is still remains elusive. We hope that our results can give a new pulse to solve this problem.

\section{ACKNOWLEDGMENT}

The author thanks to Dr. Valery Mishchenko for the fruitful discussions. This work was supported in part by a grant from the Ministry of Education of Belarus and a grant from the MOST project (an EU funded project). 


\section{REFERENCES}

[1] P. Ball, Life's Matrix: A Biography of Water, Berkeley: University of California Press, 2001, ch.1, 2.

[2] M. J. Mottl, B. T. Glazer, R. I. Kaiser, and K. J. Meech, Water and astrobiology," Chemie der Erde - Geochemistry, no. 67, pp. 253-282, Dec. 2007

[3] J. M. Zheng and G. N. Pollack, Łong-range forces extending from polymer-gel surfaces", Phys. Rev. E Stat. Nonlinear Soft Matter Phys., vol. 68, no. 3, pp. 031408-031414, Sep. 2003.

[4] G. N. Pollack, X. Figueroa, and Q. Zhao, Molecules, water, and radiant energy: New clues for the origin of life," Int. J. Mol. Sci., vol. 10, no. 4, pp. 1419-1429, 2009.

[5] B. Chai and G. H. Pollack, Solute-free interfacial zones in polar liquids", J. Phys. Chem. B, vol. 114, no. 16, pp. 5371-5375, Apr. 2010.

[6] E. Del Giudice, P. R. Spinetti, and A. Tedeschi, Water dynamics at the roots of the metamorphosis in living organisms," Water, vol. 2, no. 3, pp. 566-586, Sep. 2010.

[7] R. M. Davidson, A. Lauritzen, and S. Seneff, Biological water dynamics and entropy: A biophysical origin of cancer and other diseases", Entropy, vol. 15, no. 9, pp. 3822-3876, Sep. 2013.

[8] F. T. Hong, Magnetic field effects on biomolecules, cells, and living organisms," Biosystems, vol. 36, no. 3, pp. 187-229, 1995.

[9] P. E. Kovacs, R. L. Valentine, and P. J. J. Alvarez, The effect of static magnetic fields on biological systems: Implications for enhanced biodegradation," Critical Rev. Envir. Sci. Techn., vol. 27 no. 4, pp. 319-382, 1997.

[10] M. E. Maffei, Magnetic field effects on plant growth, development, and evolution," Front. Plant Sci., vol. 5, pp. 1-15, Sep. 2014.

[11] X. F. Pang and B. Deng, The changes of macroscopic features and microscopic structures of water under influence of magnetic field," Physica. B, vol. 403, no. 19-20, pp. 3571-3577, Oct. 2008.

[12] X. F. Pang, B. B. Deng, and B. Tang, fnfluences of magnetic field on macroscopic properties of water," Mod. Phys. Lett. B, vol. 26, no. 11, p. 1250069, 2012.

[13] A. Yadollahpour, S. Rashidi, Z. Rezaee, and M. Jalilifar, Magnetic water treatment in environmental management: A review of the recent advances and future perspectives," Current World Environment, vol. 9, no. 3, pp. 1008-1016, Dec. 2014.

[14] A. R. Davis and Jr. W. C. Rawls, Magnetism and Its Effects on the Living System, Kansas, U.S.A.: Exposition Press, 1974.

[15] J. Jung, B. Sanji, S. Godbole, and S. Sofer, Biodegradation of phenol: A comparative study with and without applying magnetic fields," J. Chem. Tech. Biotechnol., vol. 56, no. 1, pp. 73-76, 1993.

[16] J. Jung and S. Sofer, Enhancement of phenol biodegradation by south magnetic field exposure," J. Chem. Tech. Biotechnol., vol. 70, no. 3, pp. 299-303, 1997.

[17] V. W. Adamkiewicz, C. Bassous, D. Morency, P. Lorrain, and J. L. Lepage, Magnetic response in cultures of Streptococcus mutans ATCC-27607," Exp. Biol., vol. 46, no. 3, pp. 127-132, 1987.

[18] C. M. Bertea, R. Narayana, C. Agliassa, C. T. Rodgers, and M. E. Maffei, Geomagnetic field (Gmf) and plant evolution: investigating the effects of $\mathrm{Gmf}$ reversal on Arabidopsis thaliana development and gene expression", J. Vis. Exp., vol. 30, no. 105, p. e53286, Nov. 2015.

[19] V. Nimmi and G. Madhu, Effect of pre-sowing treatment with permanent magnetic field on germination and growth of chilli (Capsicum annum L.)," Int. Agrophysics, vol. 23, no. 2, pp. 19598, June 2009.

[20] A. Trappier, P. Lorio, and L. P. Johnson, Evolving perspectives on the exposure risks from magnetic fields", J. Nat. Med. Assoc., vol. 82, no. 9, pp. 621-624, Sep. 1990.
[21] S. Ghodbane, A. Lahbib, M. Sakly, and H. Abdelmelek, Bioeffects of static magnetic fields: Oxidative stress, genotoxic effects, and cancer studies, " Biomed Res Int., vol. 2013, Aug. 2013.

[22] C. Vergallo, M. Ahmadi, H. Mobasheri, and L. Dini, fmpact of Inhomogeneous Static Magnetic Field (31.7-232.0 mT) Exposure on Human Neuroblastoma SH-SY5Y Cells during Cisplatin Administration," PLoS ONE, vol. 9, no. 11, P. e113530, Nov. 2014.

[23] Y. Li and P. Héroux, Extra-low-frequency magnetic fields alter cancer cells through metabolic restriction," Electromagnetic Biology and Medicine, vol. 33, no. 4, pp. 264-275, 2014.

[24] M. R. Hinman, -Eomparative effect of positive and negative static magnetic fields on heart rate and blood pressure in healthy adults," Clin Rehabil., vol. 16, no. 6, pp. 669-674, Sep. 2002.

[25] H. N. Mayrovitz and E. E. Groseclose, Effects of a static magnetic field of either polarity on skin microcirculation," Microvasc. Res., vol. 69, no. 1-2, pp. 24-27, Jan. 2005.

[26] A. Occhipinti, A. De Santis, and M. E. Maffei, Magnetoreception an unavoidable step for plant evolution?" Trends Plant Sci., vol. 19, no. 1, pp. 1-4, Jan. 2014.

[27] A. Shrivastava, K K. Mahajan, V. Kalra, and K. S. Negi, Effects of electromagnetic forces of earth on human biological system," Indian J. Prev. Soc. Med., vol. 40, no. 3-4, pp. 162-167, 2009.

[28] L. Holysz, A. Szczes, and E. Chibowski, Effects of static magnetic field on water and electrolyte solutions," J. Colloid Interface Sci., vol. 316, no. 2, pp. 996-1002, Dec. 2007.

[29] J. Nakagawa, N. Hirota, K. Kitazawa, and M. Shoda, Magnetic field enhancement of water vaporization", J. Appl. Phys., vol. 86, no. 5, pp. 2923-2925, Sep. 1999.

[30] Yun-Zhu, et al., Evaporation Rate of Water as a Function of a Magnetic Field and Field Gradient", Int. J. Mol. Sci., vol. 13, pp. 16916-16928, Dec. 2012.

[31] Yu. S. Sarkisov, et al., The temperature responses of water and aqueous solutions to the external magnetic field effect," Tomsk State University Journal of Chemistry, no. 2, pp. 20-29, 2015.

[32] E. Ben-Jacob, Fowards a Systemic View of Water as the Fabric of Life", Water: A Multidisciplinary Research J., vol. 2, pp. 8-10, Jan. 2010.

[33] Shalatonin, Biophysical properties of liquid water exposed to EM radio frequency radiation," in Electromagnetic Radiation, S. O. Bashir, Ed., InTech, 2012, ch. 12, pp. 269-288.

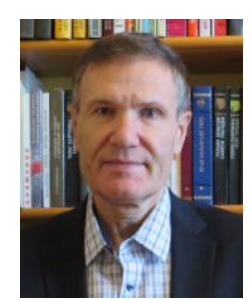

Valery Shalatonin was born in Belarus in 1950. Graduated from the Minsk University of Radiotechnics in 1972. Since 1972 he has been with Science Research Institute of Facilities for Automation where he worked as a Researcher and later a Senior Researcher. In 1986 he received Ph.D. in Radio Systems Including Microwaves Engineering and Technology from the Minsk University of Radiotechnics. Since 1992 he works as an Associate Professor, Department of Telecommunication Systems, since 1996 - Head of a Research laboratory at the Belarusian State University of Informatics and Radioelectronics.

His fields of research \& professional interests are biological coherence and response to external stimuli; biological oscillatory phenomena; Large-scale, fractal behaviour of oscillatory systems; physical mechanisms and biological properties of water following exposure to non-thermal electromagnetic (magnetic) fields; biomedical effects of weak electromagnetic fields; protection from the mobile phones electromagnetic radiation.

Dr. Shalatonin is a member of the Belarusian Physical Association, a Fulbright Scholar (2017). The author and co-author more than 100 publications and patents. 